\title{
Molecular Modeling of the Structural and Dynamical Changes in Calcium Channel TRPV5 Induced by the African-Specific A563T Variation
}

\author{
Supporting information \\ Lingyun Wang ${ }^{1}$, Ross Holmes ${ }^{2}$, Ji-Bin Peng ${ }^{1,2 *}$ \\ ${ }^{1}$ Division of Nephrology, Department of Medicine, Nephrology Research and Training Center; \\ ${ }^{2}$ Department of Urology, \\ University of Alabama at Birmingham, Birmingham, Alabama 35294, United States
}

* To whom correspondence should be addressed:

Division of Nephrology, Department of Medicine University of Alabama at Birmingham

ZRB 634, 1900 University Boulevard,

Birmingham, AL 35294-0006

Email: jpeng@uab.edu 


\section{SUPPORTING INFORMATION - TEXT}

Part I. Detailed description of the correlation in Figure 6. Compared to TRPV5-A563, TRPV5-T563 has less correlated motions (regions I and II in the white boxes of Figure 6) and anticorrelated motions (regions III, IV, V, VI, and VII in the black boxes of Figure 6) between residues in different monomers. Region I shows the correlated motions between residues 566595 (second half of TM6 and TRP domain) of monomer 1 and monomer 3. Region II shows the correlated motions between residues 504-577 (the second half of TM5, selective filter, and TM6) of monomer 4 and residues 514-556 (loop between TM5 and TM6, including the selective filter) of monomer 2. Region III shows the anticorrelated motions between residues 504-577 (the second half of TM5, selective filter, and TM6) of monomer 1 and residues 473-515 (TM4-TM5 linker and TM5) of monomer 2. Region IV shows the anticorrelated motions between residues 493-545 (TM5 and the selective filter) of monomer 3 and residues 473-595 of monomer 1.

Region V shows the anticorrelated motions between residues 526-580 (pore helix, selective filter, and TM6) of monomer 3 and residues 473-548 (TM4-TM5 linker, TM5, pore helix and selective filter) of monomer 2. Region VI shows the anticorrelated motions between residues 504-577 (the second half of TM5, selective filter and TM6) of monomer 4 and residues 514-556 (loop between TM5 and TM6 containing selective filter) of monomer 1. Region VII shows the anticorrelated motions between residues 504-580 (the second half of TM5, the selective filter, and TM6) of monomer 4 and residues 473-556 (M4-TM5 linker, TM5, pore helix, and the selective filter) of monomer 2. 


\section{Part II. Simulation results for the alternative model.}

We also evaluated the structural and dynamical changes of A563T variation using the alternative model based on the alignment shown in Figure S1b. The parameters and conditions for the original models were kept in the simulations for the alternative models. Similar to the original models, the simulations were performed for $400 \mathrm{~ns}$ and the data from the last $200 \mathrm{~ns}$ were used in the analyses. The alternative models had significantly higher RMSD values than the original models $(3.89 \pm 0.14 \AA$ vs. $3.26 \pm 0.18 \AA$ for TRPV5-A563 and $3.92 \pm 0.15 \AA$ vs. $3.06 \pm$ $0.10 \AA$ for TRPV5-T563, Figure S7b). This indicated that the alternative models had larger structural deviations from the initial structure than the original ones.

Results of secondary structure analysis indicated that the alternative models also exhibited less helix occupancy than the original ones in the modeled region of TRPV5 (61.52\% vs. $66.05 \%$ for TRPV5-A563) (Figure S8). The helix occupancy for the TRPV1 template is $74.36 \%$, thus the original model is closer to the template than the alternative one in $\alpha$ helix structure. The structural change for the alternative models came from the alternative alignment (Figure S1) where a gap in the pore helix of TRPV5 (residue 527-538) was introduced in order to align ${ }^{643} \mathrm{GMGD}^{646}$, the selective filter of TRPV1, with ${ }^{541}$ IDAP $^{544}$ of TRPV5. As a result, the helix occupancy of the pore helix in the alternative models decreased significantly (51.62\% vs. 90.52\% for TRPV5-A563, shaded regions in Figure S8).

Despite the differences between the alternative and original models, similar changes caused by $\mathrm{A} 563 \mathrm{~T}$ variation were also observed in the alternative model. The finding that $\mathrm{A} / \mathrm{T} 563$ interacts with V540 in the original model (Figure 2) suggests that D542 in the alternative model, which corresponds to V540 in the original model, may interact with A/T563. Indeed, a very low level of interaction occurred between T563 and D542 but not between A563 and D542 in the 
simulation (Figure S9a). The low level of interaction between A/T563 and D542 in the alternative models as compared to the high level of interaction between A/T563 and V540 in the original models is likely due to the negatively charged D542 residue being shifted towards the center of the pore in the presence of positive charged $\mathrm{Ca}^{2+}$ ions (Figure S9b and S9c, $\mathrm{Ca}^{2+}$ ions not shown). Instead of V540 in the original models, filter residues P544 and I541 made contact with $\mathrm{A} / \mathrm{T} 563$ (Figure S9b and S9c), and the interaction between I541 and residue 563 was increased in TRPV5-T563. The interaction between A/T563 and D542 is likely minor as compared to other stronger interactions in affecting the structure and dynamics of the TRPV5 selective filter in the alternative model.

Similar to the original model, the monomers of TRPV5-A563 exhibited more collective motion than those of TRPV5-T563 (Figure S10), indicating the A563T variation results in more stabilized dynamics among monomers. Although the difference was not as robust as observed in the original models, the surface electrostatic negative potential of TRPV5-T563 was reduced compared to that of TRPV5-A563 (left panels in Figure S11). In addition, a difference in potential of the inner pore was not observed between A563 and T563 in the alternative model (right panels in Figure S11). Furthermore, the RMSF values for residues around D542 and around A/T563 (Figure S12), and helix occupancy around residues 563 (shaded in Figure S13) were not significantly different between TRPV5-A563 and TRPV5-T563 in the alternative models. 


\section{SUPPORTING INFORMATION - FIGURES}
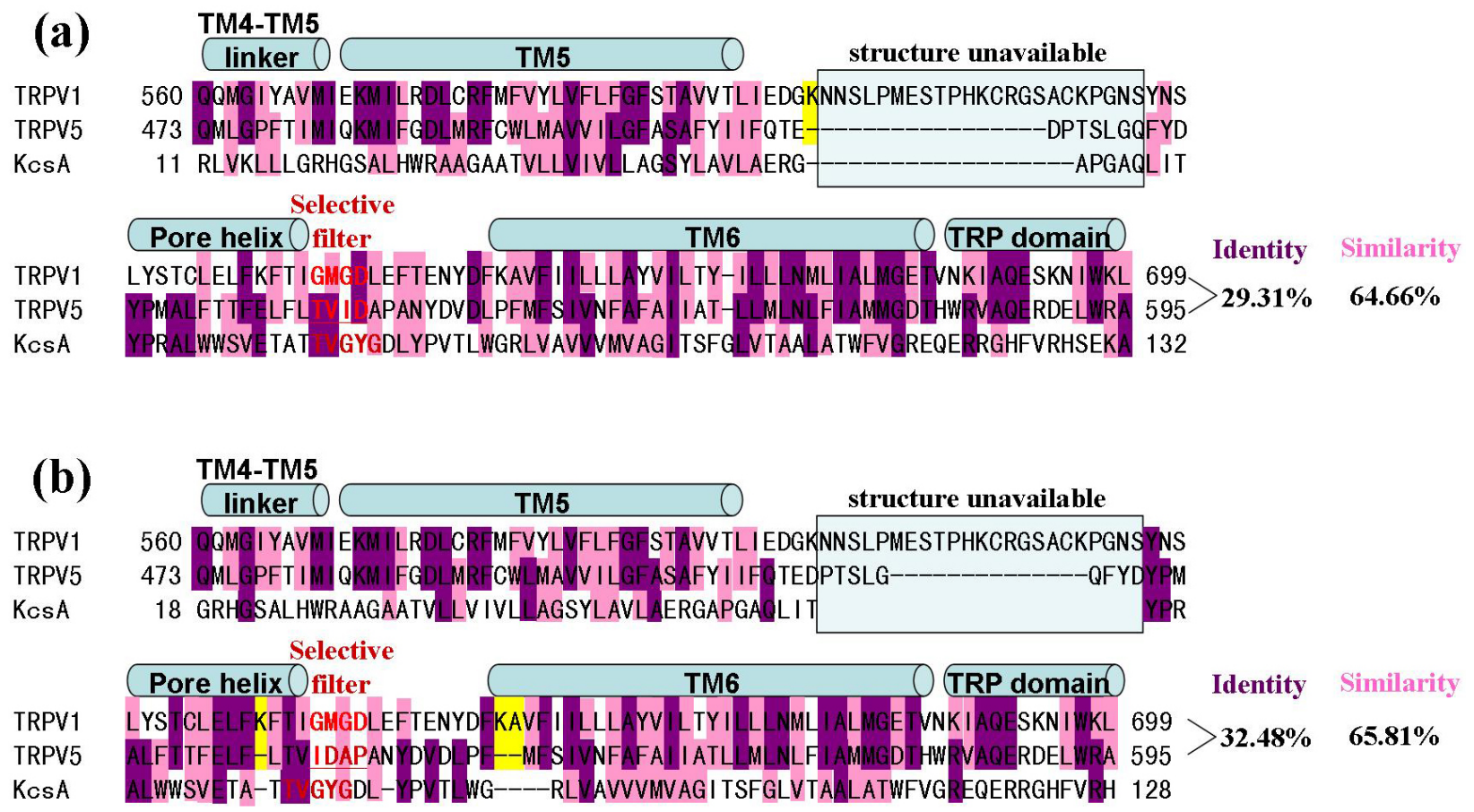

Figure S1. Sequence alignments of TRPV5 (model) with TRPV1 (template). (a) The selective filter of TRPV1 ( ${ }^{643} \mathrm{GMGD}^{646}$ ) was aligned with ${ }^{539}$ TVID $^{542}$ of TRPV5 by ClustalW2 program as done by Liao et al. (1). (b) The selective filter of TRPV1 $\left({ }^{643} \mathrm{GMGD}^{646}\right)$ was aligned with ${ }^{541}$ IDAP $^{544}$ of TRPV5 similar to the alignments by Dodier et al. (2) and Yeh et al. (3). The domains in TRPV1 are indicated. The TRPV1 structural data are not available in the shaded region and this region was not modeled in TRPV5. 
(a) ${ }^{539} \mathrm{TVID}^{542}:{ }^{643} \mathbf{G M G D}^{646}(\mathrm{TRPV} 1)$

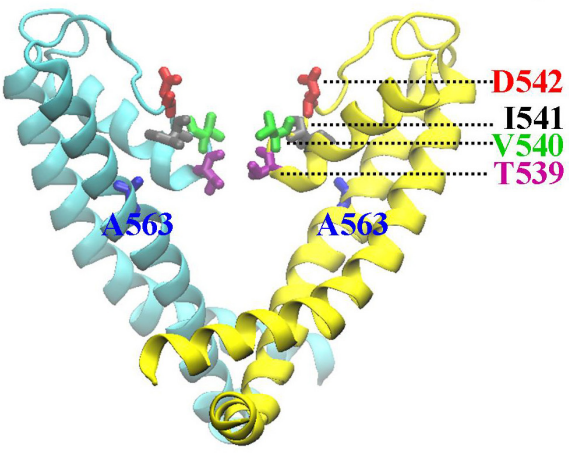

(c)



(e) Ca $\mathrm{ab}$ (PDB:4MS2)

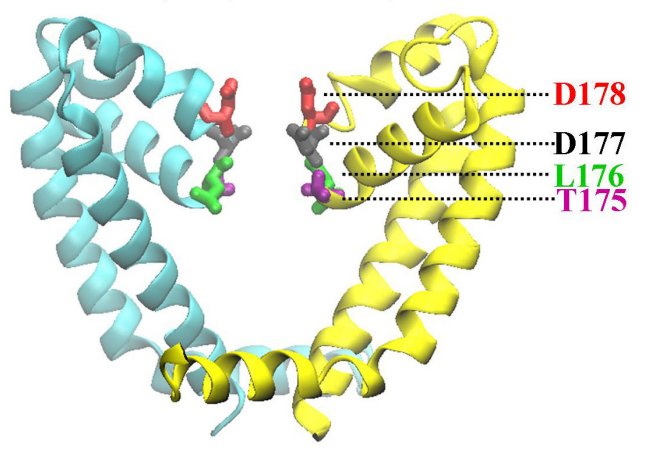

(b) ${ }^{541}$ IDAP $^{544}:{ }^{643}$ GMGD $^{646}(\mathrm{TRPV} 1)$

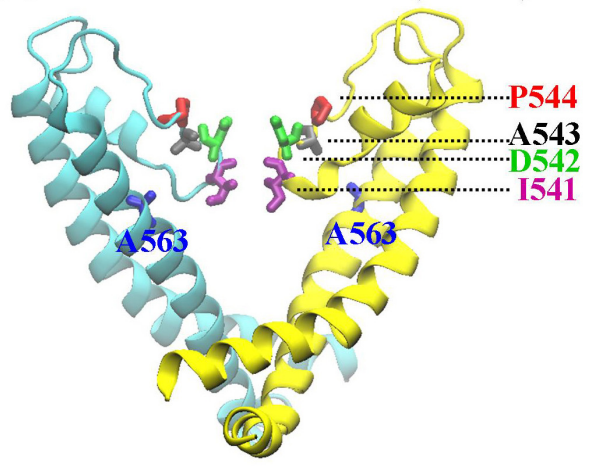

(d) KcsA (PDB: 1J95)



Figure S2. Two models of TRPV5 based on the two alignments in Figure S1 and the existing models of TRPV1, KcsA, and $\mathrm{Ca}_{\mathbf{v}} \mathrm{Ab}$ for comparison. (a) Modeled structure based on the alignment in Figure S1a. (b) Modeled structure based on the alignment in Figure S1b. (c) PDB structure (3J5P) of TRPV1. (d) PDB structure (1J95) of KcsA (a K ${ }^{+}$channel). (e) PDB structure (4MS2) of $\mathrm{Ca}_{\mathrm{v}} \mathrm{Ab}\left(\mathrm{a} \mathrm{Ca}^{2+}\right.$ channel). Filter residues in each model are indicated in colors. Residue D542 (key residue for $\mathrm{Ca}^{2+}$ permeation) is in the entrance of TRPV5 in Figure (a), but it locates further into the pore in Figure (b). 


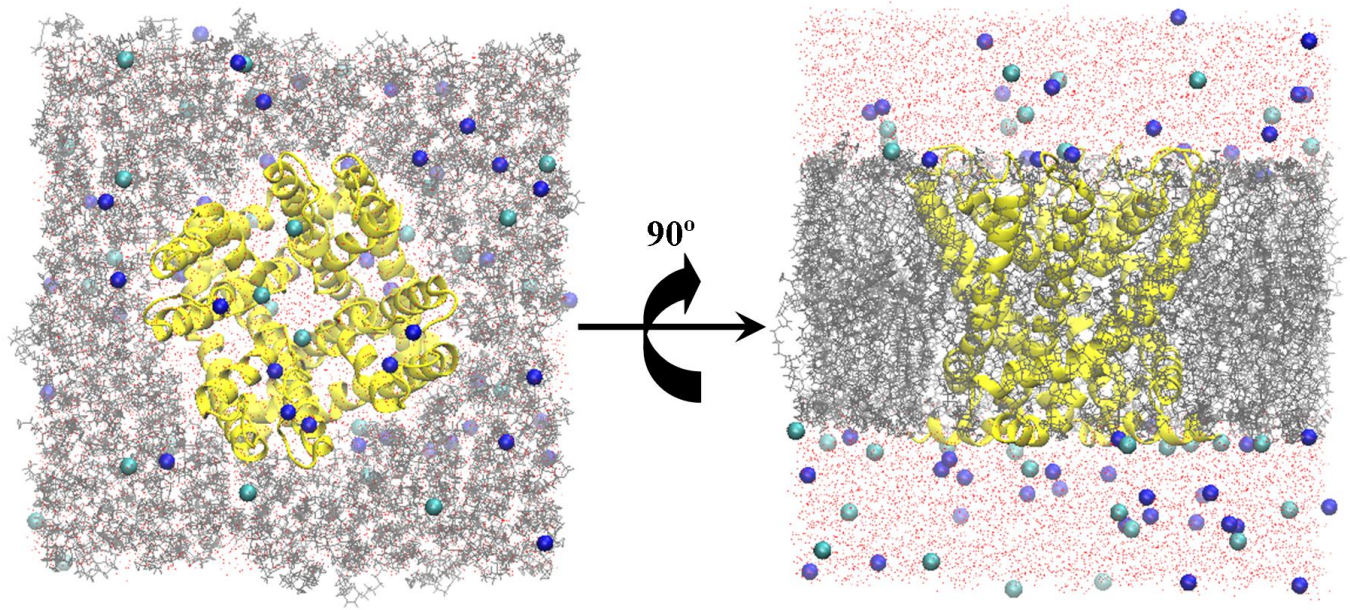

Figure S3. TRPV5 pore (residue 473-595) in a POPC (1-palmitoyl-2-oleoyl-sn-glycero-3phosphocholine) membrane-like environment. Left panel, top view; right panel, side view. TRPV5 is shown in yellow, POPC lipids in gray, $\mathrm{Na}^{+}$in blue, $\mathrm{Cl}^{-}$in cyan, and water molecules are shown as red dots. 


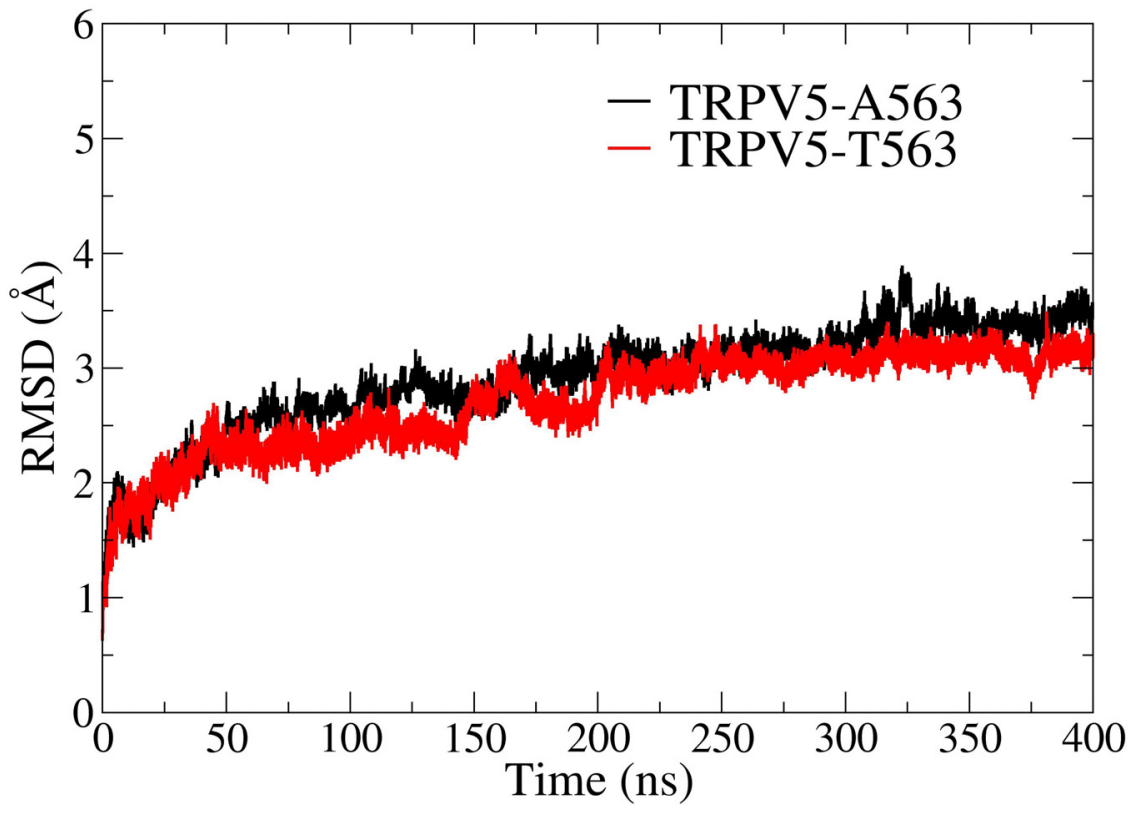

Figure S4. Equilibration of TRPV5. RMSD results indicate that the two TRPV5 systems were equilibrated after $200 \mathrm{~ns}$. 

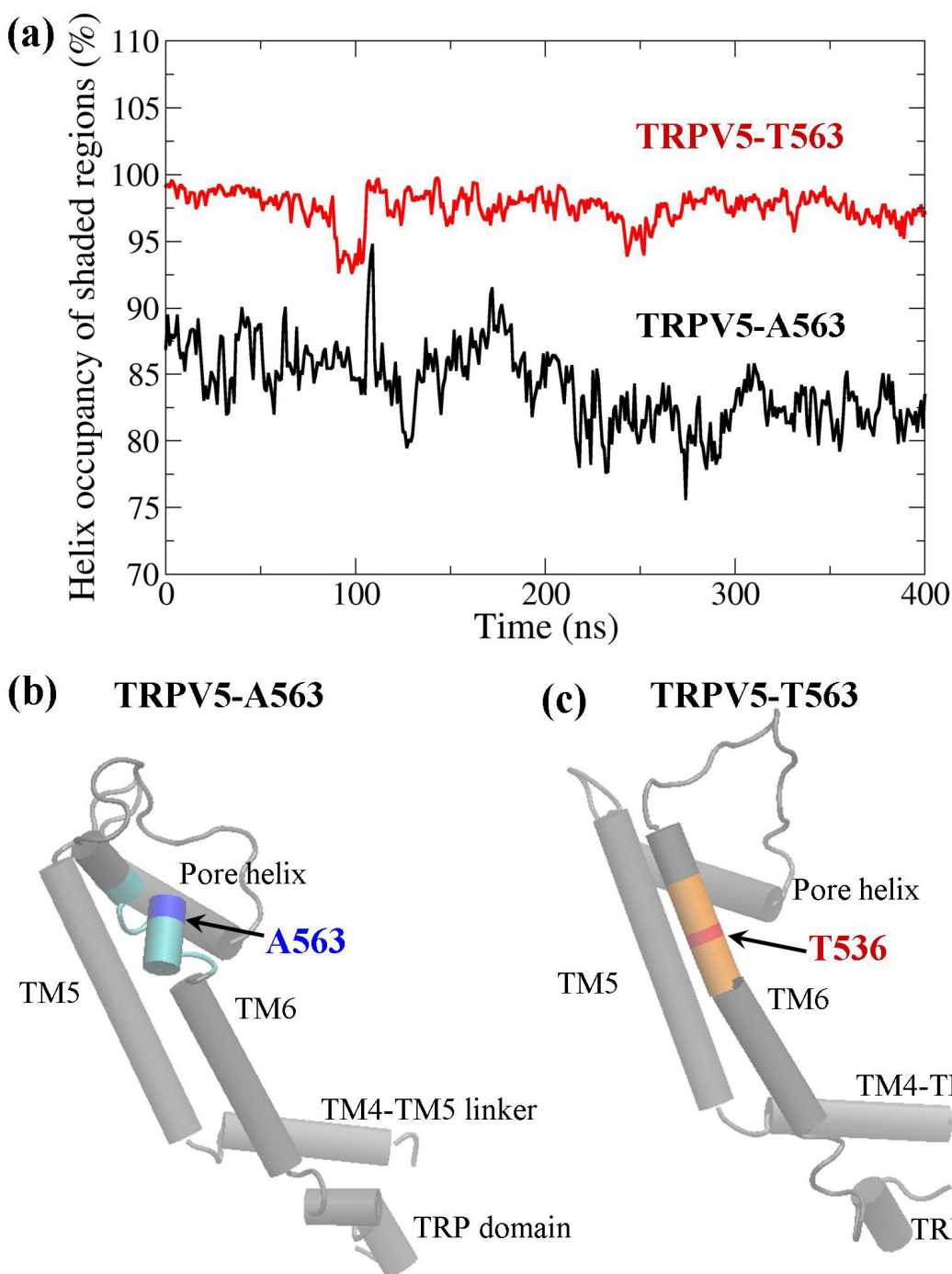

(c) TRPV5-T563

Figure S5. Change of helix occupancy for the regions around residue 563. (a) Helix occupancy (averaged every $1 \mathrm{~ns}$ ) during the course of simulation. (b) and (c) show the representative structure of monomer 3 in TRPV5-A563 and TRPV5-T563, respectively. The regions around residue 563 are shown in color: cyan for TRPV5-A563 and orange for TRPV5T563. 
(a)

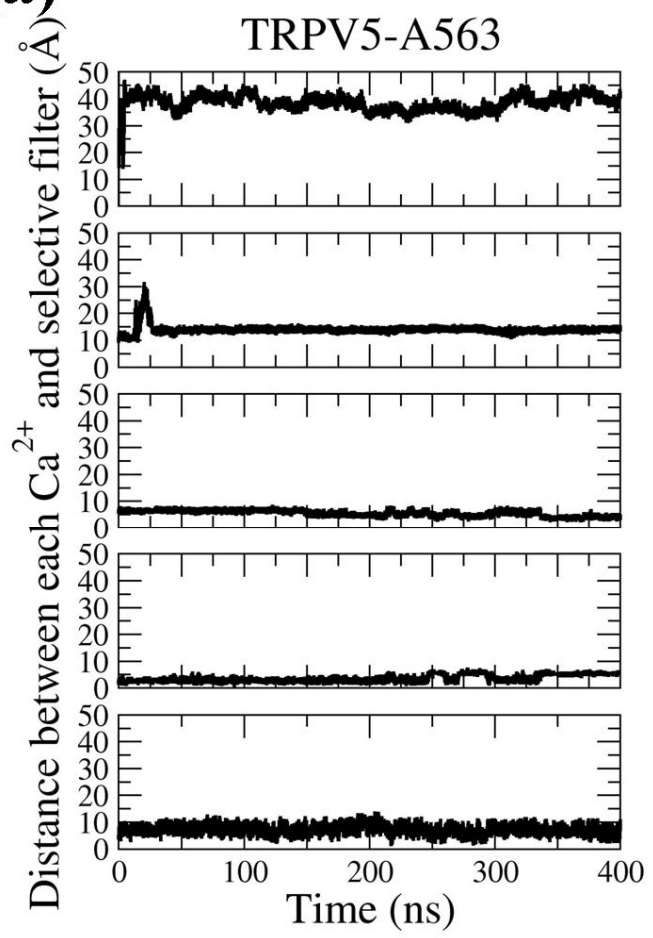

TRPV5-T563
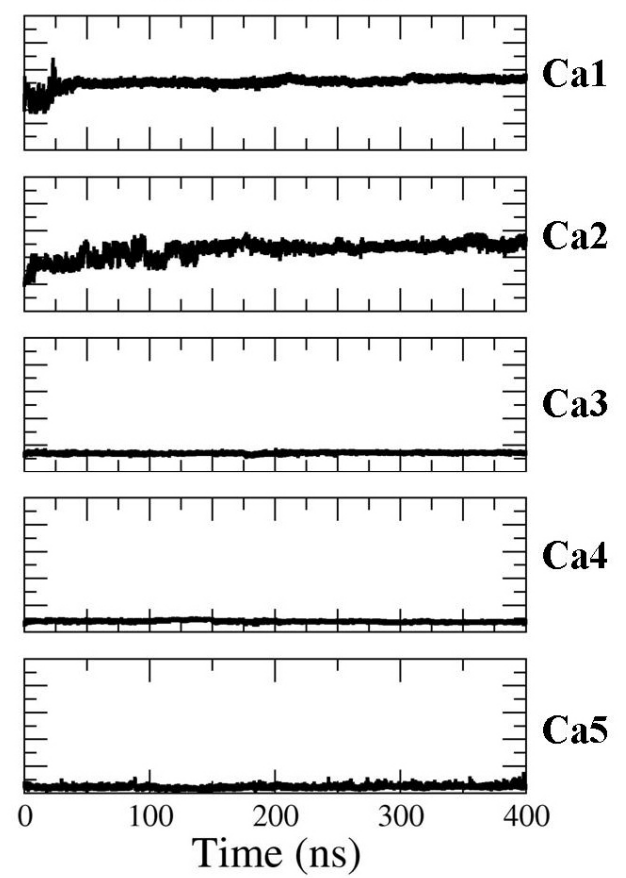

(b)

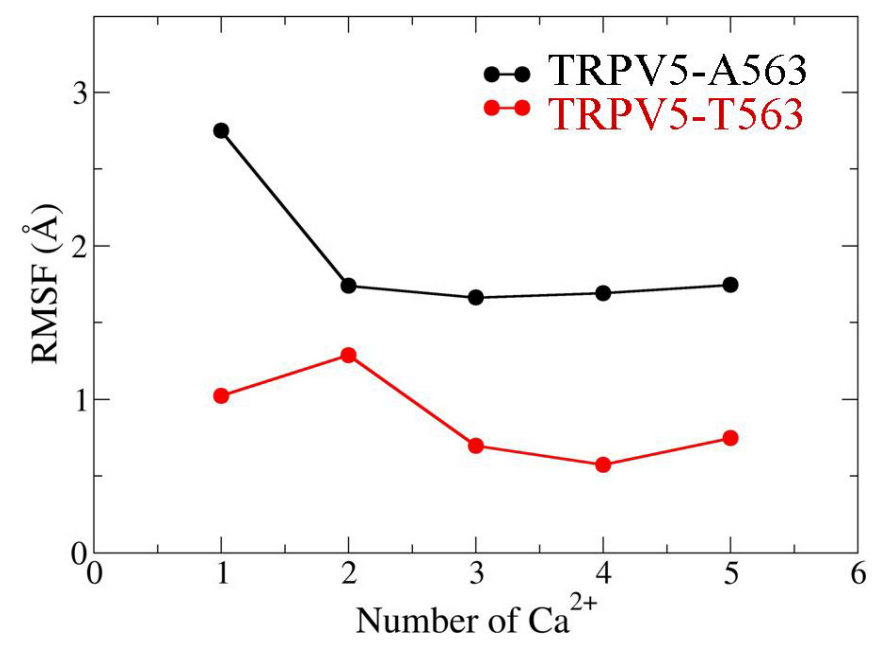

Figure S6. Dynamical change of $\mathrm{Ca}^{2+}$ ions. (a) The distance between each $\mathrm{Ca}^{2+}$ ion and the center of selective filter. The distance between $\mathrm{Ca}^{2+}$ ion and the center of selective filter was within $10 \AA$ for $\mathrm{Ca} 3, \mathrm{Ca} 4$, and $\mathrm{Ca} 5$, whereas that for $\mathrm{Ca} 1$ and $\mathrm{Ca} 2$ exceeded $10 \AA$, indicating that $\mathrm{Ca} 1$ and $\mathrm{Ca} 2$ moved away from their initial sites. (b) Comparison of $\mathrm{RMSF}$ for $\mathrm{Ca}^{2+}$ ions in TRPV5-A563 and TRPV5-T563. The RMSF values of $\mathrm{Ca}^{2+}$ ions in TRPV5-T563 were lower than those in TRPV5-A563. 


\section{(a) Alternative model}


Figure S7. Root mean square deviation (RMSD) comparisons between TRPV5 variants. (a)

Time-dependent RMSD for the alternative models. (b) RMSD comparison between the original models and the alternative models. The RMSD values for the alternative models are larger than those for the original ones. The standard deviation was calculated based on the statistically independent periods of MD simulation trajectories as described in Statistical Methods. * indicates that the difference is statistically significant based on the mean and standard deviation of the analyzed variable (Student's $t$ test, $p<0.05$ ). 


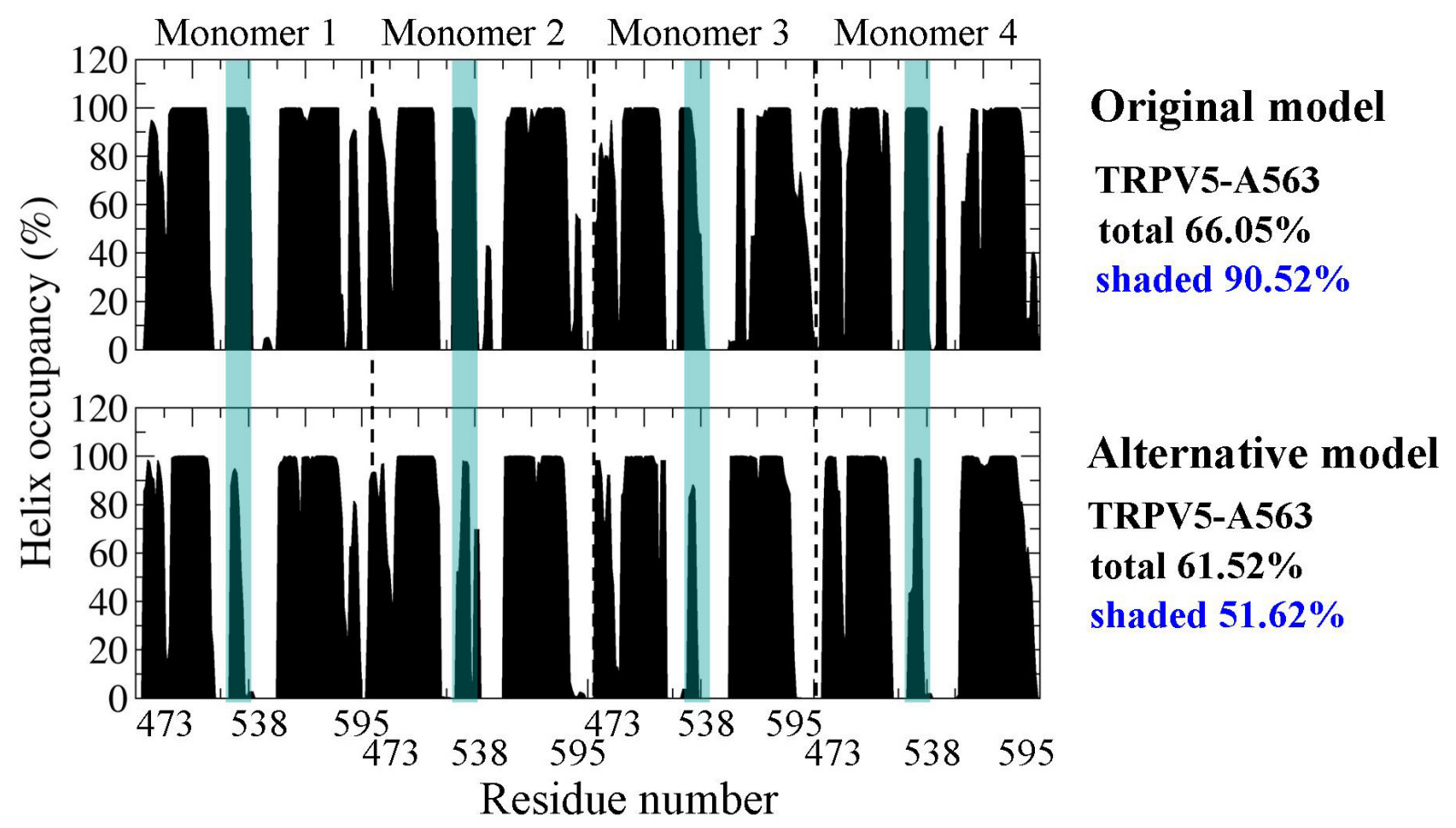

Figure S8. Secondary structure comparison between the original and alternative model for TRPV5-A563 system. The alternative model has less helix occupancy than the original one, especially for the pore helix (shaded regions: residues 527-538). 


\section{Alternative model}

(a) TRPV5-A563

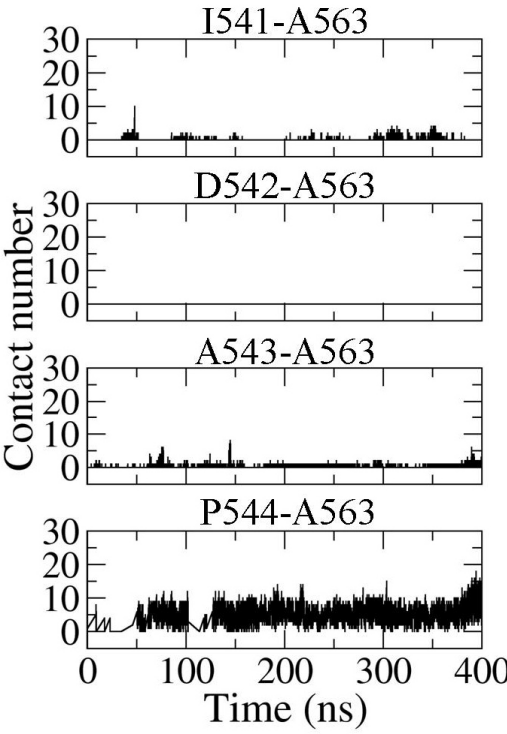

TRPV5-T563

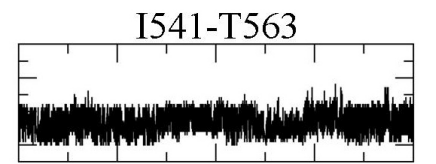

D542-T563

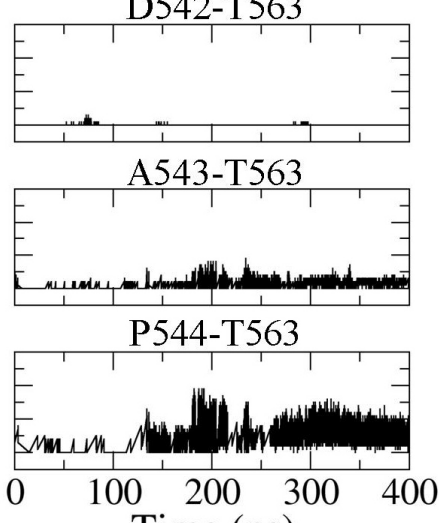

Time (ns)

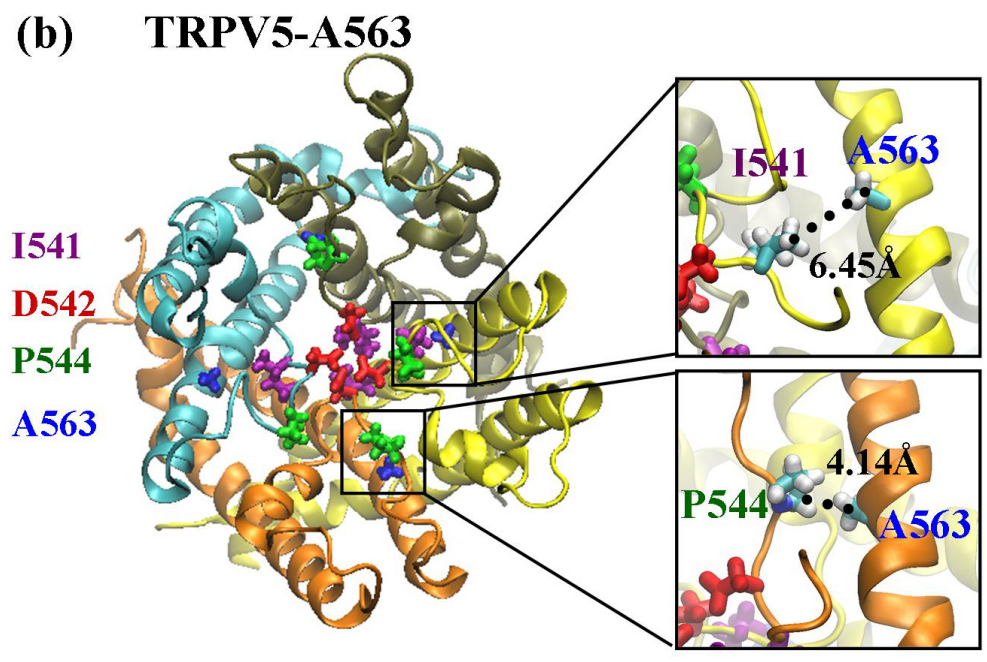

(c) TRPV5-T563

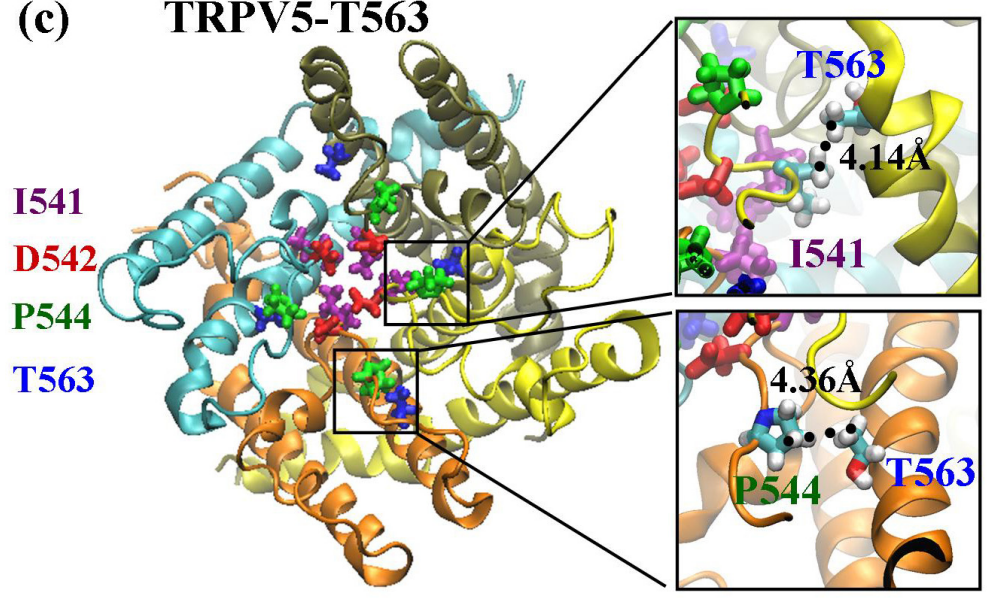


Figure S9. Interaction between filter residues and residue 563 for the alternative models. (a) Time-dependent contact number between each filter residue and residue 563 in TRPV5-A563 (left) and TRPV5-T563 (right). (b) and (c), representative structures exhibiting the interaction between filter residues (P544 and I541) and A/T563. The view of the enlarged figure is oriented to clearly show the interaction between residues. Distance between residue 563 and P544/I541 is also shown. For the interacting residues in the enlarged figures, carbon atoms are labeled in cyan and hydrogen atoms are labeled in white. 


\section{Alternative model}

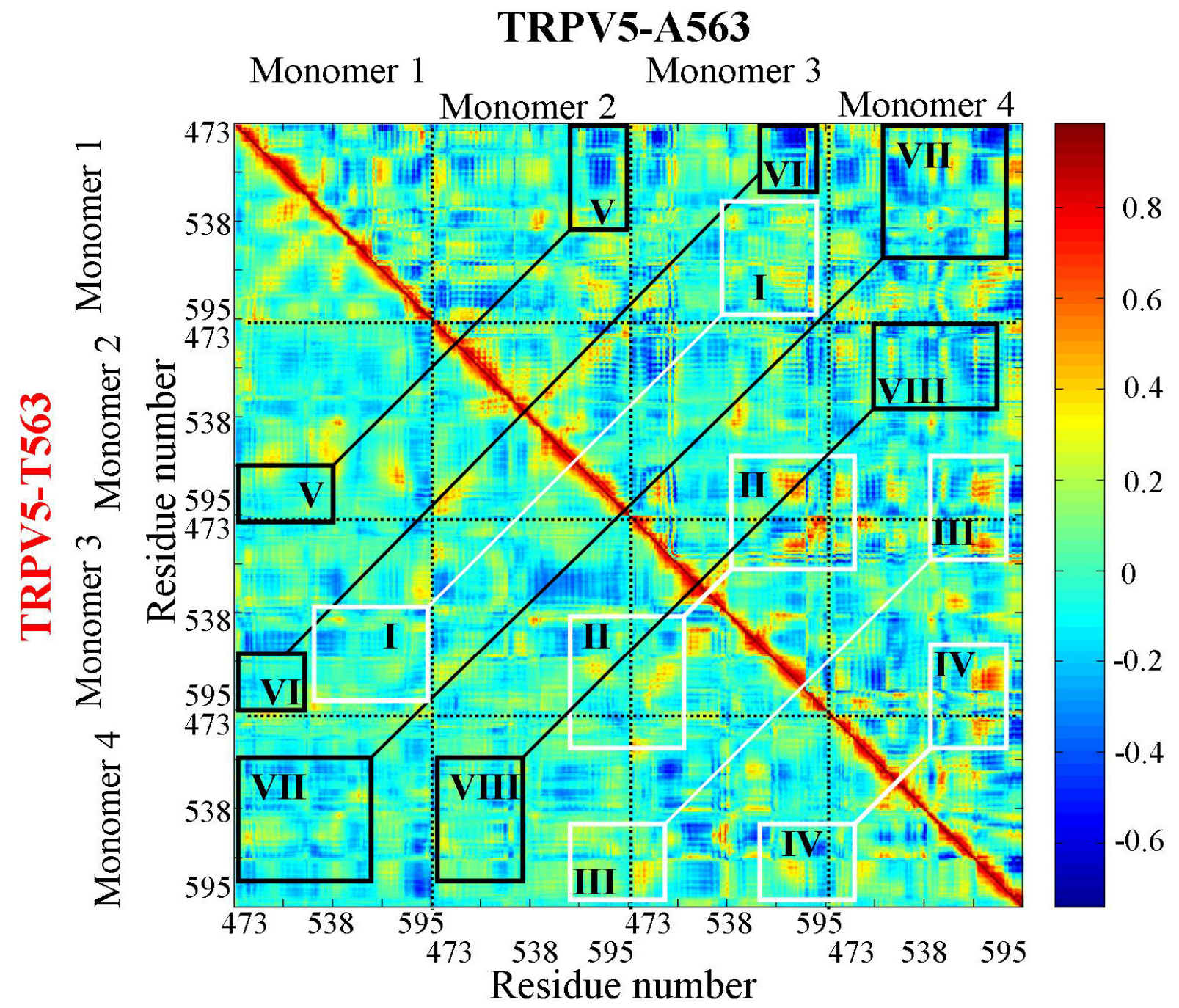

Figure 10. Dynamical cross-correlation maps (DCCM) for the comparison of the degree of correlated motion of the residues in TRPV5-A563 (top right) and TRPV5-T563 (bottom left) for the alternative model. The red color indicates the correlation between residues, whereas the blue color indicates the anticorrelation between residues. The changed regions of positive correlation are represented in white boxes, whereas the changed regions of anticorrelation in black boxes. 


\section{Alternative model}

(a)

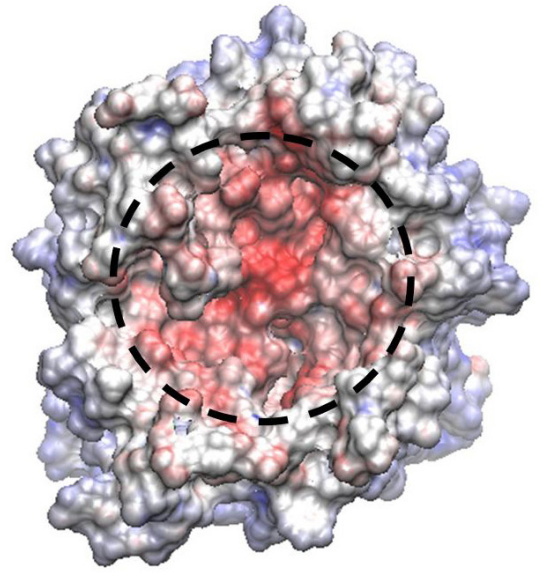

(c)

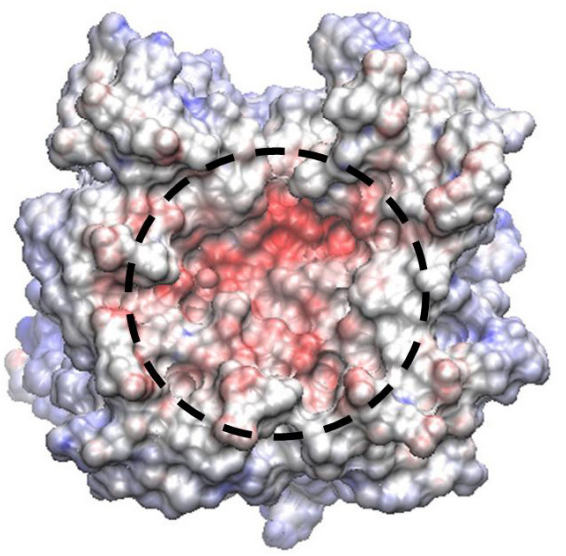

(b)

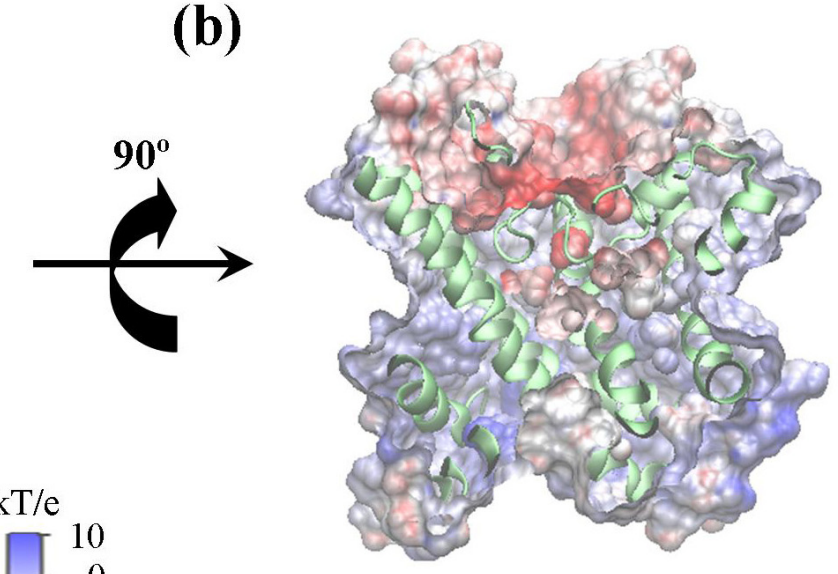

(d)

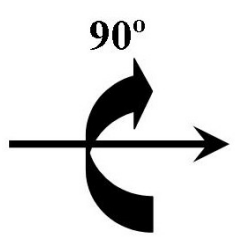

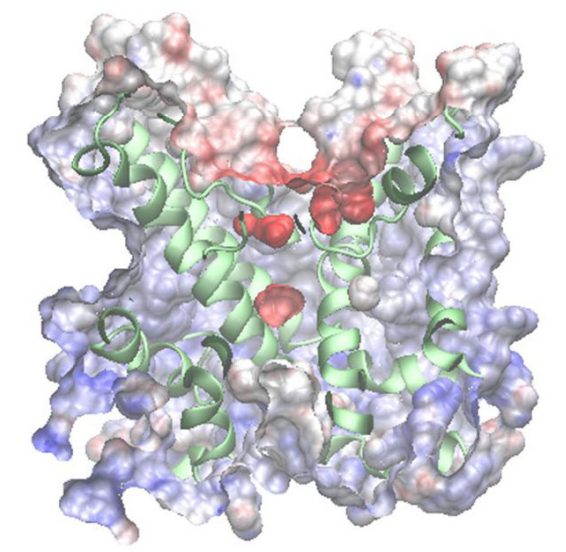

Figure S11. Comparison of the electrostatic potential between TRPV5-A563 (a-b) and TRPV5-T563 (c-d) for alternative models. Top view of the surface electrostatic potential for TRPV5 pore is shown on the left whereas side view of the electrostatic potential projected on the inner pore's van der Waals surface is shown on the right. Dashed circles indicate the outer surface of channel pore. 


\section{Alternative model}

(a) -TRPV5-A563

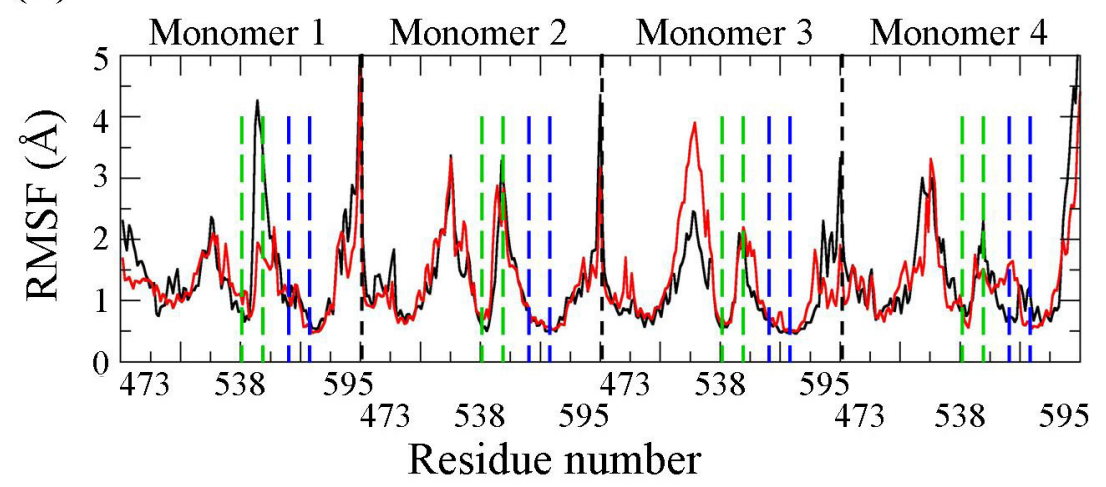

(b)


(c)

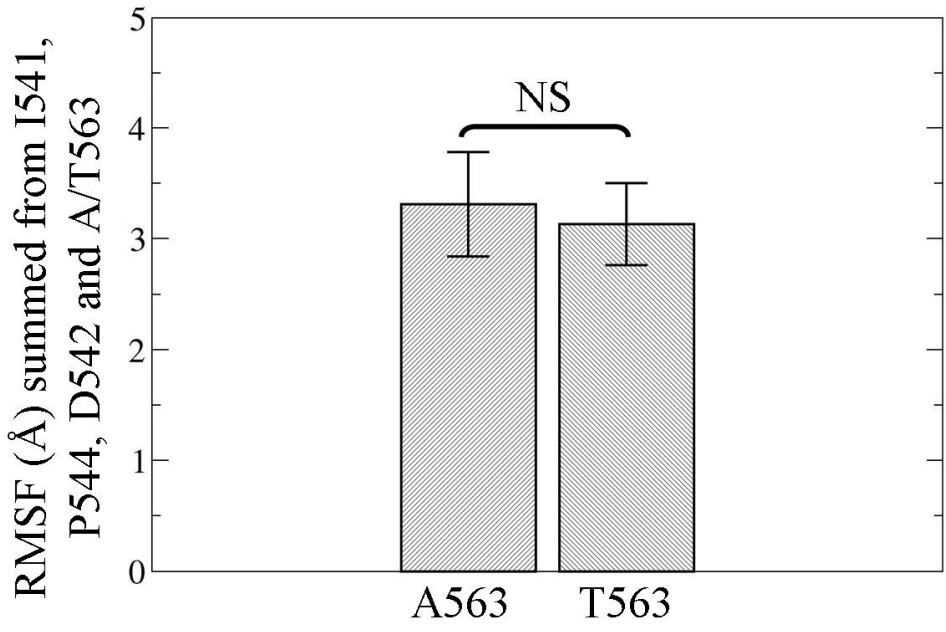


Figure S12. RMSF comparisons between TRPV5 variants for the alternative models. (a) RMSF comparison for the four monomers of TRPV5. Monomers are separated by black dashed lines. The regions between green dashed lines and between blue dashed lines are zoomed in and shown in the left and right panels of Figure b, respectively. (b) RMSF comparison for residues around D542 (left panel) and around residue 563 (right panel). The black dashed lines indicate the positions of I541, D542 and P544 in the left panel, and A/T563 in the right panel, respectively. (c) The RMSF values summed from I541, D542, P544 and residue 563. The RMSF sum between TRPV5-A563 and TRPV5-T563 is not significantly (NS) different. 


\section{Alternative model}

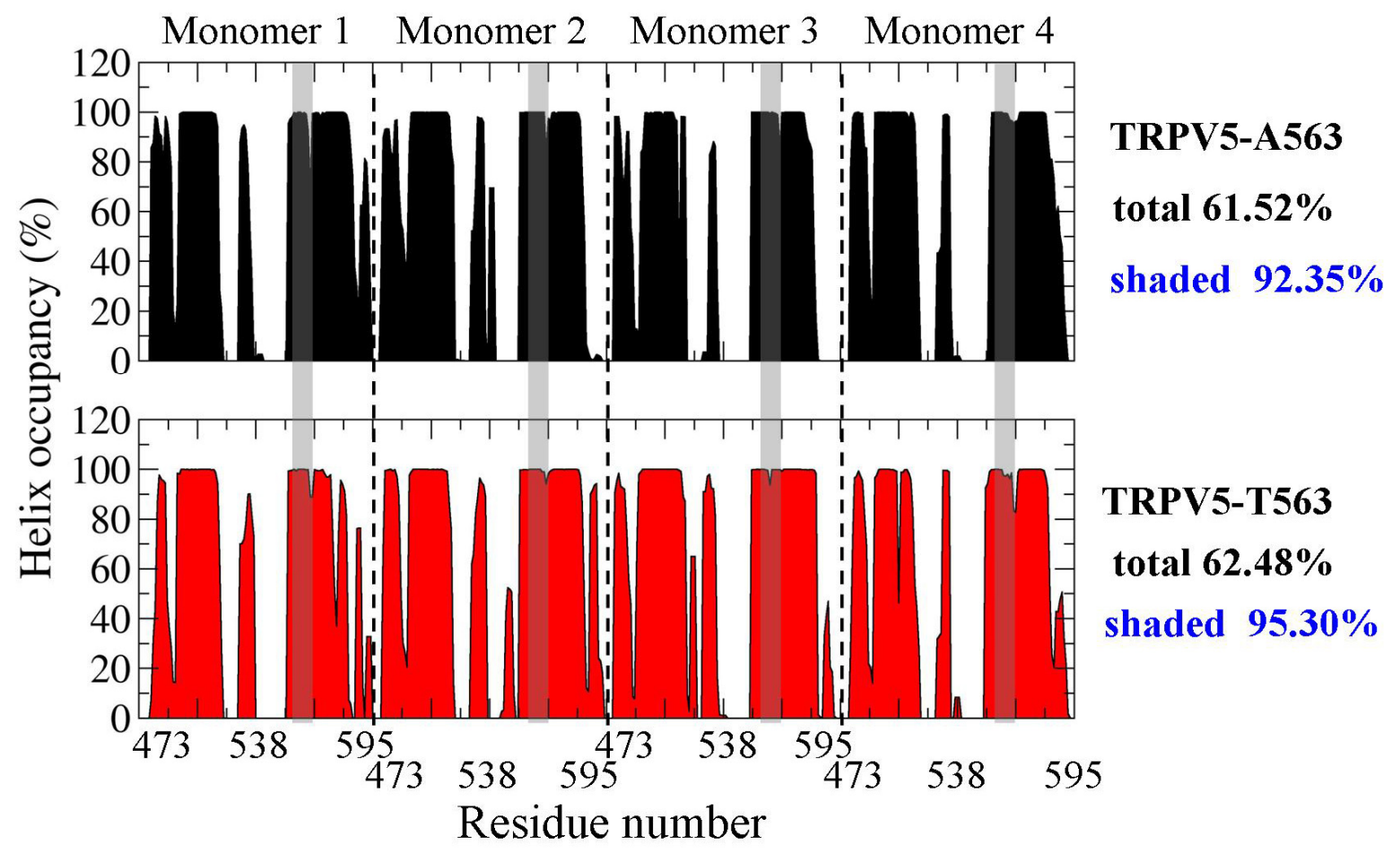

Figure S13. Residues with $\alpha$-helix occupancy in TRPV5-A563 and TRPV5-T563 for the alternative models. The overall helix percentage in the region covering amino-acid residues 473-595 in each of the four monomers of TRPV5 was calculated and expressed as the overall helicity in that region divided by the total residue number of that region. The region shaded represents residues 559-567 around A563 (upper panel) or T563 (lower panel) in each TRPV5 monomer. 
Table S1. Evaluation of the TRPV5 models.

\begin{tabular}{|c|c|c|c|c|c|c|c|c|c|}
\hline Evaluation & MODELLER & $E R R A T$ & $\begin{array}{r}P \\
\text { (total }\end{array}$ & $\begin{array}{l}\text { ROCHEC } \\
11 \text { evalua }\end{array}$ & $\begin{array}{l}K \\
\text { ions) }\end{array}$ & $\begin{array}{r}W \\
\text { (total }\end{array}$ & $\begin{array}{l}\boldsymbol{H A T C H E} \\
66 \text { evalua }\end{array}$ & $\begin{array}{l}\text { CK } \\
\text { ions) }\end{array}$ & VERIFY3D \\
\hline PDB & DOPE & & PASS & Warning & Error & PASS & Warning & Error & \\
\hline $3 J 5 P$ & & 90.82 & 4 & 3 & 4 & 41 & 19 & 6 & $24.79 \%$ \\
\hline model-1 & -73334.99 & 81.14 & 6 & 3 & 2 & 47 & 14 & 5 & $21.55 \%$ \\
\hline model-2 & -73268.59 & 73.90 & 6 & 2 & 3 & 47 & 14 & 5 & $21.55 \%$ \\
\hline model-3 & -73200.00 & 73.68 & 7 & 1 & 3 & 46 & 15 & 5 & $20.69 \%$ \\
\hline model-4 & -73195.17 & 72.81 & 6 & 3 & 2 & 46 & 15 & 5 & $21.55 \%$ \\
\hline model-5 & -73163.47 & 76.32 & 6 & 3 & 2 & 47 & 14 & 5 & $13.79 \%$ \\
\hline model-6 & -73151.35 & 79.83 & 6 & 2 & 3 & 47 & 14 & 5 & $15.52 \%$ \\
\hline model-7 & -73143.38 & 72.37 & 6 & 3 & 2 & 47 & 14 & 5 & $18.10 \%$ \\
\hline model-8 & -73126.32 & 76.32 & 6 & 2 & 3 & 45 & 16 & 5 & $25.00 \%$ \\
\hline model-9 & -73090.18 & 72.15 & 7 & 3 & 1 & 46 & 15 & 5 & $18.97 \%$ \\
\hline model-10 & -73053.84 & 73.68 & 6 & 3 & 2 & 45 & 16 & 5 & $15.52 \%$ \\
\hline
\end{tabular}

The first 10 structures sorted by DOPE energy from MODELLER were further assessed by different statistical methods, including ERRAT, PEOCHECK, WHAT_CHECK, and VERIFY3D. The structure with the lowest DOPE energy, highest ERRAT value, and fewest errors checked by PROCHECK and WATCHECK was chosen as the best model. The PDB files for the pore structure of TRPV5 models are provided in separate PDB files as a part of Supporting Information. 
Table S2. Averaged RMSD values ( $\AA$ ) for each monomer of TRPV5

\begin{tabular}{l|cc}
\hline System & TRPV5-A563 & TRPV5-T563 \\
\hline Monomers & $2.24 \pm 0.14$ & $2.36 \pm 0.10$ \\
Monomer 1 & $2.85 \pm 0.13$ & $2.31 \pm 0.14$ \\
Monomer 2 & $3.18 \pm 0.35$ & $3.62 \pm 0.22$ \\
Monomer 4 & $2.81 \pm 0.28$ & $2.64 \pm 0.23$ \\
\hline
\end{tabular}




\section{REFERENCES FOR SUPPORTING INFORMATION:}

1. Liao, M., E. Cao, D. Julius, and Y. Cheng. 2013. Structure of the TRPV1 ion channel determined by electro cyo-microscopy. Nature 504:107-112.

2. Dodier, Y., U. Banderali, H. Klein, O. Topalak, O. Dafi, M. Simoes, G. Bernatchez, R. Sauve, and L. Parent. 2004. Outer pore topology of the ECaC-TRPV5 channel by cysteine scan mutagenesis. J. Biol. Chem. 279:6853-6862.

3. Yeh, B., Y. K. Kim, W. Jabbar, and C. Huang. 2005. Conformational changes of pore helix coupled to gating of TRPV5 by protons. EMBO J. 24:3224-3234. 